\title{
Productivity Efficiency and Leaf Reddening in Bt Cotton (Gossypium hirsutum L.) as Influenced by SSNM Based Nutrition for Targeted Yields
}

\author{
Vinayak Hosamani $^{1 *}$, B.M. Chittapur ${ }^{2}$, Mallikarjun ${ }^{3}$, A.S. Halepyati ${ }^{4}$, \\ Satyanarayana Rao ${ }^{5}$, M.B. Patil ${ }^{6}$, N.L. Rajesh $^{7}$ and Venkatesh Hosamani ${ }^{8}$ \\ ${ }^{1}$ P2 BSF, Nagenahally, Kunigal, Central Silk Board, Bangalore/ UAS, \\ Raichur, Karnataka, India \\ ${ }^{2}$ Directorate of extension, University of Agricultural Sciences, Raichur, Karnataka, India \\ ${ }^{3} e-S A P$, Project, University of Agricultural Sciences, \\ Raichur, Karnataka, India \\ ${ }^{4}$ University of Agricultural Sciences, Raichur, Karnataka, India \\ ${ }^{5}$ PI \& Head, Research Institute on Organic Farming, MARS, UAS, Raichur, Karnataka, India \\ ${ }^{6}$ AEEC, Koppal, UAS, Raichur, Karnataka, India \\ ${ }^{7}$ (SS\&AC), COA, UAS, Raichur, Karnataka, India \\ ${ }^{8}$ Entomology, COH, Munirabad-Koppal, Karnataka, India \\ *Corresponding author
}

\section{A B S T R A C T}

The field experiment on cotton productivity and leaf reddening as influenced by nutrition management for targeted yield was conducted during growing seasons of 2014-15 and 2015-16 at College of Agriculture Farm, Raichur, Karnataka on medium deep black soil

Keywords

Bt cotton, SSNM and RDF, Cotton, Productivity efficiency

Article Info

Accepted:

22 July 2018

Available Online:

10 August 2018 under irrigation. Three yield targets $\left(3,4\right.$ and $5 \mathrm{t}$ kapas yield $\left.\mathrm{ha}^{-1}\right)$ based site specific nutrient management (SSNM) along with four leaf reddening management (LRM) treatments (S1 - Vermicompost @ $2.5 \mathrm{t} \mathrm{ha}^{-1}$ in seed line, S2 - S1 + MgSO $10 \mathrm{~kg} \mathrm{ha}^{-1}$ in seed line, $\mathrm{S} 3-\mathrm{S} 1+\mathrm{MgSO}_{4} 25 \mathrm{~kg} \mathrm{ha}^{-1}$ in seed line, and S4 - $\mathrm{MgSO}_{4} 25 \mathrm{~kg} \mathrm{ha}^{-1}$ in seed line + foliar nutrition of $1 \% \mathrm{MgSO}_{4}+19: 19: 19+1 \% \mathrm{KNO}_{3}$ trice during flowering, boll development and boll bursting stages) besides recommended control were tested using RCBD. SSNM for $5 \mathrm{t} \mathrm{ha}^{-1}$ yield target and supplementary nutrition of MgSO4 both to soil and to foliage and foliar application of major nutrients (19:19:19 and $\left.\mathrm{KNO}_{3}\right)(\mathrm{S} 4)$ recorded higher productivity efficiency ( 0.44 on pooled basis) and lower leaf reddening indices throughout $(0.23,0.37,0.68$ and 1.10 at $90,105,120$ and 135 DAS, respectively pooled basis), whereas lower productivity efficiency with higher reddening indices were recorded with lowering of nutrition in commensuration with lower yield targets; $3 \mathrm{t} \mathrm{ha}^{-1}$ coupled with vermicompost alone $\left(\mathrm{M}_{1} \mathrm{~S}_{1}\right)$ had higher indices $(0.60,1.16,1.53$ and 2.02 at $90,105,120$ and at 135 DAS respectively on pooled basis) throughout among all.

\section{Introduction}

Green Revolution and succeeding many other technology led revolutions in agriculture from later part of $20^{\text {th }}$ century helped to enhance productivity to cope up with the burgeoning population and industrial demands in the country. However, the repercussions of 
changing climate, unabated degradation of natural resources and continued faulty agricultural practices are still threatening the success and the production sustainability achieved so far and challenging future targets. Nevertheless, the gap between the potential productivity or best productivity of lead countries or of progressive farmers and the present general average yield in the country offers scope for betterment agronomically. Cotton being an important crop is not an exception for such technological evolution. Cotton (Gossypium spp.), 'the king of fibers' also popularly known as 'the white gold' enjoys a pre-eminent position amongst cash crops in the world and in India as well. It is the nature's most precious gift to the mankind, contributed by the genus Gossypium to clothe the people all over the world. In Karnataka, cotton occupies an area of 4.64 lakh ha with a production of 21.00 lakh bales and productivity of $769 \mathrm{~kg} \mathrm{ha}^{-1}$ (CAB, 2016). In the northern dry zones (Zone 2 and 3) covering partly the Tungabhadra and the Upper Krishna command areas (TBP and UKP), $B t$ cotton is intensively cultivated on black clayey soils under irrigation. The area under cotton crop in these command areas driven by market price and high crop productivity has been increasing rapidly over the past decade. Of late, however, there is gradual decline in productivity in the region for varied reasons as elsewhere leading to distress among farmers. There is great discontent in different quarters with the cultivars as some varieties are becoming vulnerable to boll worm (mostly due to spurious seed/F2 seed) and/or to many physiological disorders and, hence, yield below par (Venkateshwaralu, 2002) besides producing poor quality fibre as reported in Maharashtra and Gujarat (Hebbar and Mayee, 2011). Balanced nutrition based on soil test ensures efficient use of both applied and soil available nutrients and helps in sustaining the soil and crop productivity. The productivity efficiency could be considered as an effective major for screening cultivars/ LRM techniques or any other production interventions to evaluate performance, resilience or susceptibility for leaf reddening in cotton under any agro climatic condition. This index could be considered more effective because it extrapolates leaf reddening index to the whole photosynthetic surface from flowering till final picking as a contributing factor towards yield. It is advantageous over either leaf reddening index or leaf area index alone because it considers ultimate photosyenthtically effective leaf area during the reproductive cycle, while leaf reddening index is a fixed stage variation while reddening is dynamic and dependent on prevailing rhizosphere and crop microclimate conditions, and leaf area indicates total leaf area irrespective of its colour mosaic. Whereas, productivity efficiency is a single value relating yield versus potential photosynthetic leaf area during reproductive period. In this context, the present investigation Productivity enhancement and management of leaf reddening in Bt cotton (Gossypium hirsutum L.) under TBP irrigation command was planned and executed during the growing seasons of 2014 and 2015 under irrigation.

\section{Materials and Methods}

Experiment was carried out at Agricultural College Farm, University of Agricultural Sciences, Raichur, and Karnataka during growing seasons of 2014-15 and 2015-16 under irrigation. The experiment consisted of three main plot treatments (SSNM based nutrition for 3, 4 and $5 \mathrm{t} \mathrm{ha}^{-1}$ seed cotton - M13 ) and four sub plot treatments (nutrient supplementation to manage leaf reddening malady (LRM): S1 - Vermicompost @ $2.5 \mathrm{t}$ ha-1 in seed line, $\mathrm{S} 2-\mathrm{S} 1+\mathrm{MgSO}_{4} 10 \mathrm{~kg} \mathrm{ha}^{-1}$ in seed line, $\mathrm{S} 3-\mathrm{S} 1+\mathrm{MgSO}_{4} 25 \mathrm{~kg} \mathrm{ha}^{-1}$ in seed line and $\mathrm{S} 4-\mathrm{MgSO}_{4} 25 \mathrm{~kg} \mathrm{ha}^{-1}$ in seed 
line + foliar nutrition of $1 \% \mathrm{MgSO}_{4}+19: 19: 19$ $+1 \% \mathrm{KNO}_{3}$ thrice during flowering, boll development and boll bursting stages) along with recommended fertilizer practice (RDF) as outside control for comparison $(3 \times 4+1)$. For the yield targets fertilizers were applied based on the soil test and crop requirement as per SSNM (IPNI) (Table 1). In control the recommended doses of fertilizers were applied $\left(150 \mathrm{~N}, 75 \mathrm{P}_{2} \mathrm{O}_{5}\right.$ and $\left.75 \mathrm{~K} \mathrm{~kg} \mathrm{ha}^{-1}\right)$. The Productivity efficiency $\left(\mathrm{kg} \mathrm{ha}{ }^{-1} \mathrm{dm}^{-2}\right.$ day $\left.^{-1}\right)$ could be considered as an effective major for screening cultivars/ LRM techniques or any other production interventions to evaluate performance, resilience or susceptibility for leaf reddening in cotton under any agro climatic condition. It is new ratio developed and used in the study to evaluate productivity as related to photosynthetically active green surface as follows

$\begin{aligned} & \text { Productivity efficiency } \\ & \text { Yield (kg/ha) }\end{aligned}$
$L A(\operatorname{dm} 2) X L R I X$ duration(days)

(PE) =

Where, LA- Leaf area $\left(\mathrm{dm}^{-1}\right)$

LRI-Leaf reddening index

Leaf reddening was recorded for quantitative estimation of degree of leaf reddening, observations were recorded at 90, 105, 120 and 135 DAS as outlined by Dastur et al., (1952). The number of leaves showing signs of reddening, partly or wholly were divided into five categories on the visual observations. At 60 DAS, Bt cotton plants were not exhibiting the symptoms of leaf reddening, hence not recorded.

\section{Results and Discussion}

\section{Productivity efficiency (kg ha $\left.{ }^{-1} \mathrm{dm}^{-2} \mathrm{day}^{-1}\right)$}

Productivity efficiency, a new tool developed for assessing productivity as a consequence of green leaf surface varied significantly due to SSNM based major nutrients application for varied yield targets (Table 2). Productivity efficiency was the maximum $(0.51$ on pooled basis) with yield target of $5 \mathrm{tha}^{-1}\left(\mathrm{M}_{3}\right)$ during both the years of experimentation and on pooled basis. The productivity ratio linearly and significantly decreased as the yield target decreased and recorded the lower productivity efficiency (0.33) with the yield target of $3 \mathrm{t}$

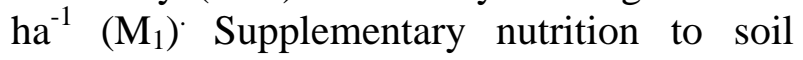
and/or foliage comprising major and micro nutrients for the leaf reddening management (LRM) also influenced cotton productivity efficiency significantly during the course experimentation. Treatment comprising $\mathrm{MgSO}_{4}$ supply both to soil and to foliage in addition to $19: 19: 19$ and $\mathrm{KNO}_{3}\left(\mathrm{~S}_{4}\right)$ fared better among the different subplot treatments recording overall higher productivity ratio ( 0.44 on pooled basis) during both the years and on pooled basis. Other LRM treatments were intermediary or overlapping in their effects, while vermicompost alone $\left(S_{1}\right)$ had lower productivity efficiency ( 0.42 on pooled basis) among all. Further, interactions due to SSNM and nutrient supplementation for leaf reddening influenced productivity efficiency significantly, during both the years and on pooled basis as well. Overall, SSNM for yield target of $5 \mathrm{t} \mathrm{ha}^{-1}$ and supplementary nutrition of $\mathrm{MgSO}_{4}$ both to soil and to foliage and foliar application of major nutrients (19:19:19 and $\left.\mathrm{KNO}_{3}\right)\left(\mathrm{M}_{3} \mathrm{~S}_{4}\right)$ resulted in higher productivity ratio (0.52 during first year), however, different LRM practices within this yield target were comparable and were at par. $\mathrm{M}_{1} \mathrm{~S}_{1}$ with $3 \mathrm{t} \mathrm{ha}^{-1}$ target and vermicompost alone to soil had lower productivity efficiency amongst all ( 0.31 on pooled basis). Interestingly, SSNM based nutrition in combination with supplemental nutrition for leaf reddening control with yield target exceeding $4 \mathrm{t} \mathrm{ha}^{-1}$ irrespective of LRM except $\mathrm{M}_{1} \mathrm{~S}_{4}$ recorded higher productivity efficiency over control with recommended nutritional practice $(0.32$ on pooled basis) during both the years and on pooled basis as well. 


\section{Leaf Reddening Index (LRI)}

Significant differences occurred in LRI during both the years at all stages of development and on pooled basis due to SSNM based yield targets and LRM practices and their interactions (Table 2 and 3 ). The indices were significantly lower $(0.23,0.47,0.61$ and 1.10 at $90,105,120$ and 135 DAS respectively) with $5 \mathrm{t} \mathrm{ha}^{-1}$ yield target $\left(\mathrm{M}_{3}\right) ; 4 \mathrm{t} \mathrm{ha}^{-1}$ yield target fared on par while lower target of $3 \mathrm{t} \mathrm{ha}^{-}$ ${ }^{1}\left(\mathrm{M}_{1}\right)$ had higher reddening indices $(0.49$, $0.91,1.26$ and 1.56 at $90,105,120$ and 135 DAS respectively on pooled basis) among all. Similarly, application of $\mathrm{MgSO}_{4}$ both soil and foliar nutrition in addition to 19:19:19 and $\mathrm{KNO}_{3}\left(\mathrm{~S}_{4}\right)$ recorded significantly lower LRI $(0.21,0.50,0.78$ and 1.07 at $90,105,120$ and at 135 DAS, respectively on pooled basis) among LRM practices followed by integrated application of vermicompost $+\mathrm{MgSO}_{4}$ irrespective of dosage while, vermicompost alone $\left(S_{1}\right)$ had higher indices $(0.39,0.83,1.07$ and 1.52 at 90, 105, 120 and 135 DAS respectively on pooled basis). Among interaction effects due to SSNM and LRM practices, $5 \mathrm{t} \mathrm{ha}^{-1}$ yield target coupled with application of $\mathrm{MgSO}_{4}$ both to soil and to foliage and foliar application of major nutrients $\left(\mathrm{M}_{3} \mathrm{~S}_{4}\right)$ resulted in significantly lower indices throughout $(0.23,0.37,0.68$ and 1.10 at $90,105,120$ and 135 DAS, respectively pooled basis), whereas the higher reddening indices were recorded with lowering of nutrition in commensuration with lower yield targets; $3 \mathrm{t} \mathrm{ha}^{-1}$ coupled with vermicompost alone $\left(\mathrm{M}_{1} \mathrm{~S}_{1}\right)$ had higher indices $(0.60,1.16$, 1.53 and 2.02 at $90,105,120$ and at 135 DAS respectively on pooled basis) throughout among all. SSNM based nutrition in combination with supplemental nutrition for leaf reddening recorded lowest leaf reddening index compared to control $(0.83,1.43,1.73$ and 2.10 at 90, 105, 120 and at 135 DAS respectively) at all the stages of growth during both the years and on pooled basis as well.

SSNM is an approach of application of right source of fertilizer, at the right rate, right time and right place to fill the deficit between the nutrient need of a high yielding crop and nutrient supply from naturally occurring indigenous sources including soil, crop residues, manure and irrigation water. Not only SSNM has demonstrated a potential to increase crop yields and farmers profits in many crops, there is also increasing evidence of the environmental friendliness of SSNM as it focuses on balanced and crop need based nutrient application. The variations in leaf reddening index resulted in variation in productivity efficiency (Table 2 and 3 ) consequent of which yield varied significantly.

Table.1 Soil test value, ratings, nutrient requirement to achieve the target and adjusted nutrients for the I Experiment during 2014-15 and 2015-16

\begin{tabular}{|c|c|c|c|c|}
\hline \multirow{2}{*}{$\begin{array}{c}\text { Yield } \\
\text { Targets }\end{array}$} & \multicolumn{2}{|c|}{ Soil test value $\left(\mathrm{N}: \mathrm{P}_{2} \mathrm{O}_{5}: \mathrm{K}_{2} \mathrm{O} \mathrm{kg} \mathrm{ha}{ }^{-1}\right)$} & \multirow{2}{*}{$\begin{array}{c}\text { Nutrient } \\
\text { requirement } \\
\left(\mathrm{N}: \mathrm{P}_{2} \mathrm{O}_{5}: \mathrm{K}_{2} \mathrm{O} \mathrm{kg}\right. \\
\left.\mathrm{ha}^{-1}\right)\end{array}$} & \multirow{2}{*}{$\begin{array}{c}\text { Final applied } \\
\left(\mathrm{N}: \mathrm{P}_{2} \mathrm{O}_{5}: \mathrm{K}_{2} \mathrm{O}\right. \\
\left.\mathrm{kg} \mathrm{ha}^{-1}\right)\end{array}$} \\
\hline & 2014-15 & 2015-16 & & \\
\hline 3 t ha $^{-1}$ & $168: 72: 184$ & 198:74:208 & 192:84:114 & $240: 63: 114$ \\
\hline $4 \mathrm{t} \mathrm{ha}^{-1}$ & $168: 72: 184$ & 198:74:208 & $256: 112: 152$ & $316: 84: 152$ \\
\hline $5 \mathrm{t} \mathrm{ha}^{-1}$ & $168: 72: 184$ & 198:74:208 & 320:140:190 & $400: 105: 190$ \\
\hline
\end{tabular}


Table.2 Productivity efficiency $\left(\mathrm{kg} \mathrm{ha}^{-1} \mathrm{dm}^{-2} \mathrm{day}^{-1}\right)$ and Seed cotton yield $\left(\mathrm{kg} \mathrm{ha}^{-1}\right)$ of cotton influenced by SSNM based yield targets and nutrition for leaf reddening management

\begin{tabular}{|c|c|c|c|c|c|c|}
\hline \multirow[t]{2}{*}{ Treatment } & \multicolumn{3}{|c|}{$\begin{array}{l}\text { Productivity efficiency }\left(\mathrm{kg} \mathrm{ha}^{-1} \mathrm{dm}^{-2}\right. \\
\left.\text { day }^{-1}\right)\end{array}$} & \multicolumn{3}{|c|}{ Seed cotton yield $\left(\mathrm{kg} \mathrm{ha}^{-1}\right)$} \\
\hline & 2014-15 & 2015-16 & Pooled & 2014-15 & 2015-16 & Pooled \\
\hline \multicolumn{7}{|l|}{ Main plots } \\
\hline $\mathrm{M}_{1}$ & $0.34^{c}$ & $0.32^{\mathrm{c}}$ & $0.33^{c}$ & $3481^{\mathrm{c}}$ & $3483^{c}$ & $3482^{c}$ \\
\hline $\mathbf{M}_{2}$ & $0.47^{b}$ & $0.43^{b}$ & $0.45^{\mathrm{b}}$ & $4497^{b}$ & $4490^{b}$ & $4494^{\mathrm{b}}$ \\
\hline $\mathbf{M}_{3}$ & $0.53^{\mathrm{a}}$ & $0.48^{\mathrm{a}}$ & $0.51^{\mathrm{a}}$ & $5252^{a}$ & $5240^{\mathrm{a}}$ & $5246^{\mathrm{a}}$ \\
\hline S.Em土 & 0.004 & 0.010 & 0.003 & 21.2 & 20.7 & 76.9 \\
\hline \multicolumn{7}{|l|}{ Sub plots } \\
\hline $\mathbf{S}_{1}$ & $0.44^{\mathrm{b}}$ & $0.40^{\mathrm{c}}$ & $0.42^{c}$ & $4336^{\mathrm{cb}}$ & $4300^{d}$ & $4318^{b}$ \\
\hline $\mathbf{S}_{2}$ & $0.44^{b}$ & $0.41^{b}$ & $0.43^{b}$ & $4389^{\text {ba }}$ & $4377^{\mathrm{c}}$ & $4384^{\mathrm{a}}$ \\
\hline $\mathbf{S}_{3}$ & $0.44^{\mathrm{b}}$ & $0.41^{b}$ & $0.43^{\mathrm{b}}$ & $4428^{a}$ & $4438^{b}$ & $4434^{\mathrm{a}}$ \\
\hline $\mathbf{S}_{4}$ & $0.46^{\mathrm{a}}$ & $0.42^{\mathrm{a}}$ & $0.44^{\mathrm{a}}$ & $4487^{\mathrm{a}}$ & $4502^{a}$ & $4495^{\mathrm{a}}$ \\
\hline S.Em \pm & 0.004 & 0.003 & 0.003 & 20.5 & 22.3 & 40.6 \\
\hline \multicolumn{7}{|l|}{$\mathbf{M x S}$} \\
\hline $\mathbf{M}_{1} \mathbf{S}_{1}$ & $0.32^{f}$ & $0.30^{\mathrm{g}}$ & $0.31^{\mathrm{g}}$ & $3409^{f}$ & $3392^{g}$ & $3401^{i}$ \\
\hline$\overline{\mathbf{M}_{1} \mathbf{S}_{2}}$ & $0.34^{\mathrm{f}}$ & $0.33^{f}$ & $0.33^{f}$ & $3454^{\mathrm{fe}}$ & $3450^{g f}$ & $3452^{\mathrm{hi}}$ \\
\hline $\mathbf{M}_{1} \mathbf{S}_{3}$ & $0.34^{\mathrm{f}}$ & $0.32^{f}$ & $0.33^{f}$ & $3501^{\mathrm{fe}}$ & $3517^{\mathrm{gf}}$ & $3509^{\mathrm{hg}}$ \\
\hline$\overline{\mathbf{M}_{1} \mathbf{S}_{4}}$ & $0.37^{\mathrm{e}}$ & $0.33^{\mathrm{f}}$ & $0.35^{\mathrm{e}}$ & $3563^{\mathrm{e}}$ & $3573^{f}$ & $3568^{\mathrm{g}}$ \\
\hline$\overline{\mathbf{M}_{2} \mathbf{S}_{1}}$ & $0.46^{\mathrm{d}}$ & $0.42^{\mathrm{dc}}$ & $0.44^{\mathrm{d}}$ & $4426^{\mathrm{d}}$ & $4387^{\mathrm{e}}$ & $4407^{\mathrm{f}}$ \\
\hline $\mathbf{M}_{2} S_{2}$ & $0.46^{\mathrm{d}}$ & $0.43^{c}$ & $0.44^{\mathrm{d}}$ & $4487^{\mathrm{dc}}$ & $4487^{\text {ed }}$ & $4487^{\mathrm{e}}$ \\
\hline $\mathbf{M}_{2} \mathbf{S}_{3}$ & $0.47^{\mathrm{c}}$ & $0.43^{c}$ & $0.45^{\mathrm{cd}}$ & $4513^{d c}$ & $4516^{\text {ed }}$ & $4517^{\text {ed }}$ \\
\hline $\mathbf{M}_{2} \mathbf{S}_{4}$ & $0.49^{b}$ & $0.44^{c}$ & $0.47^{\mathrm{b}}$ & $4563^{c}$ & $4572^{\mathrm{d}}$ & $4568^{d}$ \\
\hline $\mathrm{M}_{3} \mathrm{~S}_{1}$ & $0.55^{\mathrm{a}}$ & $0.49^{\mathrm{a}}$ & $0.52^{\mathrm{a}}$ & $5173^{b}$ & $5122^{c}$ & $5148^{c}$ \\
\hline $\mathbf{M}_{3} \mathbf{S}_{2}$ & $0.53^{\mathrm{a}}$ & $0.49^{\mathrm{a}}$ & $0.51^{\mathrm{a}}$ & $5228^{\text {ba }}$ & $5195^{b c}$ & $5212^{\mathrm{cb}}$ \\
\hline $\mathbf{M}_{3} \mathbf{S}_{3}$ & $0.52^{\mathrm{a}}$ & $0.49^{\mathrm{a}}$ & $0.50^{\mathrm{a}}$ & $5270^{\mathrm{ba}}$ & $5280^{\mathrm{ba}}$ & $5275^{b}$ \\
\hline $\mathbf{M}_{3} \mathbf{S}_{4}$ & $0.52^{\mathrm{a}}$ & $0.47^{\text {ba }}$ & $0.50^{\mathrm{a}}$ & $5335^{\mathrm{a}}$ & $5362^{a}$ & $5349^{a}$ \\
\hline S.Em \pm & 0.01 & 0.01 & 0.01 & 34.8 & 43.7 & 86.9 \\
\hline Control & 0.33 & 0.32 & 0.32 & 2769 & 2903 & 2836 \\
\hline S.Em土 & 0.01 & 0.01 & 0.01 & 160.6 & 109.5 & 162.6 \\
\hline C.D. 0.05 & 0.02 & 0.03 & 0.03 & 468.7 & 319.5 & 474.5 \\
\hline
\end{tabular}

*means with same letters do not differ significantly under DMRT

Note: SSNM- Site Specific Nutrient Management

Main treatments: Yield Target $(M)$

$\mathrm{M}_{1}$ - SSNM for targeted yield of 3 tha $^{-1}$

$\mathrm{M}_{2}$ - SSNM for targeted yield of 4 tha $^{-1}$

$\mathrm{M}_{3}$ - SSNM for targeted yield of 5 tha $^{-1}$

Control-RDF with recommended practice
Sub treatments: Leaf reddening management $(\mathrm{S})$

$\mathbf{S}_{1^{-}}$Vermicompost @ 2.5 tha $^{-1}$ in seed line

$\mathbf{S}_{2^{-}} \mathrm{S}_{1}+\mathrm{MgSO}_{4} 10 \mathrm{kgha}^{-1}$ in seed line

$\mathrm{S}_{3}-\mathrm{S}_{1}+\mathrm{MgSO}_{4} 25 \mathrm{kgha}^{-1}$ in seed line

$\mathbf{S}_{4}-\mathrm{MgSO}_{4} 25 \mathrm{~kg} \mathrm{ha}^{-1}$ in seed line + foliar nutrition of $1 \%$

$\mathrm{MgSO}_{4}+19: 19: 19+1 \% \mathrm{KNO}_{3}$ (thrice each) 
Table.3 Leaf Reddening Index of cotton at various stages as influenced by SSNM based yield targets and nutrition for leaf reddening management

\begin{tabular}{|c|c|c|c|c|c|c|c|c|c|c|c|c|}
\hline \multirow{2}{*}{$\begin{array}{l}\text { Treat - } \\
\text { ments }\end{array}$} & \multicolumn{4}{|c|}{ 2014-15 } & \multicolumn{4}{|c|}{ 2015-16 } & \multicolumn{4}{|c|}{ Pooled } \\
\hline & $\begin{array}{c}90 \\
\text { DAS }\end{array}$ & $\begin{array}{c}105 \\
\text { DAS }\end{array}$ & $\begin{array}{c}120 \\
\text { DAS }\end{array}$ & $\begin{array}{c}135 \\
\text { DAS }\end{array}$ & $\begin{array}{c}90 \\
\text { DAS }\end{array}$ & $\begin{array}{c}105 \\
\text { DAS }\end{array}$ & $\begin{array}{c}120 \\
\text { DAS }\end{array}$ & $\begin{array}{c}135 \\
\text { DAS }\end{array}$ & $\begin{array}{c}90 \\
\text { DAS }\end{array}$ & $\begin{array}{c}105 \\
\text { DAS }\end{array}$ & $\begin{array}{c}120 \\
\text { DAS }\end{array}$ & $\begin{array}{c}135 \\
\text { DAS }\end{array}$ \\
\hline \multicolumn{13}{|c|}{ Main plots } \\
\hline $\mathrm{M}_{1}$ & $0.37^{\mathrm{a}}$ & $0.75^{\mathrm{a}}$ & $1.18^{\mathrm{a}}$ & $1.50^{\mathrm{a}}$ & $0.62^{\mathrm{a}}$ & $1.08^{\mathrm{a}}$ & $1.35^{\mathrm{a}}$ & $1.63^{\mathrm{a}}$ & $0.49^{\mathrm{a}}$ & $0.91^{\mathrm{a}}$ & $1.26^{\mathrm{a}}$ & $1.56^{\mathrm{a}}$ \\
\hline $\mathrm{M}_{2}$ & $0.22^{\mathrm{b}}$ & $0.50^{\mathrm{b}}$ & $0.70^{\mathrm{b}}$ & $1.20^{\mathrm{b}}$ & $0.34^{\mathrm{b}}$ & $0.75^{\mathrm{b}}$ & $0.93^{\mathrm{b}}$ & $1.25^{\mathrm{b}}$ & $0.26^{\mathrm{b}}$ & $0.62^{\mathrm{b}}$ & $0.81^{\mathrm{b}}$ & $1.22^{\mathrm{b}}$ \\
\hline $\mathbf{M}_{3}$ & $0.18^{b}$ & $0.37^{\mathrm{b}}$ & $0.48^{c}$ & $1.00^{\mathrm{b}}$ & $0.28^{\mathrm{b}}$ & $0.58^{c}$ & $0.74^{\mathrm{b}}$ & $1.21^{\mathrm{b}}$ & $0.23^{\mathrm{b}}$ & $0.47^{\mathrm{c}}$ & $0.61^{b}$ & $1.10^{\mathrm{b}}$ \\
\hline S.Em \pm & 0.01 & 0.03 & 0.05 & 0.06 & 0.06 & 0.04 & 0.08 & 0.09 & 0.03 & 0.03 & 0.05 & 0.05 \\
\hline \multicolumn{13}{|l|}{ Sub plots } \\
\hline$S_{1}$ & $0.31^{\mathrm{a}}$ & $0.67^{\mathrm{a}}$ & $0.98^{a}$ & $1.49^{\mathrm{a}}$ & $0.49^{\mathrm{a}}$ & $0.99^{\mathrm{a}}$ & $1.17^{\mathrm{a}}$ & $1.56^{\mathrm{a}}$ & $0.39^{\mathrm{ba}}$ & $0.83^{\mathrm{a}}$ & $1.07^{\mathrm{a}}$ & $1.52^{\mathrm{a}}$ \\
\hline $\mathbf{S}_{2}$ & $0.35^{\mathrm{b}}$ & $0.60^{\mathrm{a}}$ & $0.80^{\mathrm{b}}$ & $1.27^{\mathrm{b}}$ & $0.47^{\mathrm{a}}$ & $0.82^{\mathrm{b}}$ & $1.01^{\mathrm{b}}$ & $1.34^{\mathrm{ba}}$ & $0.40^{\mathrm{a}}$ & $0.71^{\mathrm{ba}}$ & $0.90^{\mathrm{b}}$ & $1.30^{\mathrm{b}}$ \\
\hline $\mathbf{S}_{3}$ & $0.22^{c}$ & $0.51^{\mathrm{b}}$ & $0.73^{b}$ & $1.24^{\mathrm{b}}$ & $0.39^{\mathrm{b}}$ & $0.77^{\mathrm{b}}$ & $0.92^{\mathrm{b}}$ & $1.34^{\mathrm{ba}}$ & $0.31^{\mathrm{bc}}$ & $0.64^{b}$ & $0.83^{b}$ & $1.29^{\mathrm{b}}$ \\
\hline $\mathbf{S}_{4}$ & $0.13^{\mathrm{d}}$ & $0.38^{\mathrm{c}}$ & $0.64^{b}$ & $0.93^{\mathrm{c}}$ & $0.29^{c}$ & $0.62^{c}$ & $0.91^{\mathrm{b}}$ & $1.20^{\mathrm{b}}$ & $0.21^{\mathrm{c}}$ & $0.50^{c}$ & $0.78^{b}$ & $1.07^{\mathrm{c}}$ \\
\hline S.Emt & 0.01 & 0.03 & 0.05 & 0.05 & 0.03 & 0.05 & 0.05 & 0.08 & 0.01 & 0.04 & 0.05 & 0.05 \\
\hline \multicolumn{13}{|l|}{$\mathbf{M} \times \mathrm{S}$} \\
\hline $\mathbf{M}_{1} \mathbf{S}_{1}$ & $0.47^{\mathrm{b}}$ & $1.00^{\mathrm{a}}$ & $1.47^{\mathrm{a}}$ & $2.00^{\mathrm{a}}$ & $0.74^{\mathrm{a}}$ & $1.32^{\mathrm{a}}$ & $1.60^{\mathrm{a}}$ & $2.03^{\mathrm{a}}$ & $0.60^{\mathrm{a}}$ & $1.16^{\mathrm{a}}$ & $1.53^{\mathrm{a}}$ & $2.02^{\mathrm{a}}$ \\
\hline$\overline{M_{1} S_{2}}$ & $0.60^{\mathrm{a}}$ & $0.80^{\mathrm{b}}$ & $1.13^{\mathrm{b}}$ & $1.53^{\mathrm{b}}$ & $0.76^{\mathrm{a}}$ & $1.09^{\mathrm{ba}}$ & $1.36^{\mathrm{ba}}$ & $1.62^{b}$ & $0.68^{\mathrm{a}}$ & $0.94^{\text {ba }}$ & $1.25^{\mathrm{b}}$ & $1.58^{\mathrm{b}}$ \\
\hline $\mathbf{M}_{1} \mathbf{S}_{3}$ & $0.27^{\mathrm{d}}$ & $0.73^{\mathrm{cb}}$ & $1.13^{b}$ & $1.47^{\mathrm{cb}}$ & $0.57^{\mathrm{b}}$ & $1.10^{\mathrm{ba}}$ & $1.25^{\mathrm{bc}}$ & $1.61^{\mathrm{b}}$ & $0.42^{\mathrm{b}}$ & $0.92^{\mathrm{b}}$ & $1.19^{b}$ & $1.54^{\mathrm{cb}}$ \\
\hline $\mathbf{M}_{1} \mathbf{S}_{4}$ & $0.13^{\mathrm{ef}}$ & $0.47^{\text {ed }}$ & $1.00^{\mathrm{cb}}$ & $1.00^{\mathrm{fe}}$ & $0.40^{\mathrm{dc}}$ & $0.80^{\text {dce }}$ & $1.17^{\mathrm{bc}}$ & $1.24^{\mathrm{cb}}$ & $0.27^{\mathrm{cbd}}$ & $0.63^{\mathrm{cd}}$ & $1.09^{\mathrm{cb}}$ & $1.12^{\text {ed }}$ \\
\hline$\overline{M_{2} S_{1}}$ & $0.34^{c}$ & $0.60^{\mathrm{cd}}$ & $1.00^{\mathrm{cb}}$ & $1.33^{\mathrm{cbd}}$ & $0.41^{\mathrm{c}}$ & $0.90^{b c}$ & $1.18^{\mathrm{bc}}$ & $1.36^{\mathrm{cb}}$ & $0.34^{\mathrm{cb}}$ & $0.75^{\mathrm{bc}}$ & $1.09^{\mathrm{cb}}$ & $1.35^{\mathrm{cbd}}$ \\
\hline $\mathbf{M}_{2} \mathbf{S}_{2}$ & $0.26^{\mathrm{d}}$ & $0.60^{\mathrm{cd}}$ & $0.80^{\mathrm{cd}}$ & $1.27^{\mathrm{cd}}$ & $0.38^{\mathrm{dce}}$ & $0.82^{\mathrm{dc}}$ & $0.96^{\mathrm{dc}}$ & $1.27^{\mathrm{cb}}$ & $0.29^{\mathrm{cbd}}$ & $0.71^{\text {bcd }}$ & $0.88^{\text {cd }}$ & $1.27^{\mathrm{ced}}$ \\
\hline $\mathrm{M}_{2} \mathrm{~S}_{3}$ & $0.20^{\text {ed }}$ & $0.40^{\mathrm{ef}}$ & $0.60^{\text {ed }}$ & $1.27^{\mathrm{cd}}$ & $0.35^{\mathrm{e}}$ & $0.66^{\text {dfce }}$ & $0.83^{\mathrm{d}}$ & $1.32^{\mathrm{cb}}$ & $0.28^{\mathrm{cbd}}$ & $0.53^{\mathrm{ecd}}$ & $0.71^{\mathrm{ed}}$ & $1.29^{\mathrm{cebd}}$ \\
\hline $\mathbf{M}_{2} \mathbf{S}_{4}$ & $0.17^{\mathrm{f}}$ & $0.40^{\mathrm{ef}}$ & $0.40^{\mathrm{e}}$ & $0.93^{\mathrm{fe}}$ & $0.22^{\text {dce }}$ & $0.60^{\mathrm{dfe}}$ & $0.73^{\mathrm{d}}$ & $1.04^{c}$ & $0.20^{\mathrm{d}}$ & $0.50^{\mathrm{ecd}}$ & $0.57^{\mathrm{e}}$ & $0.99^{\mathrm{e}}$ \\
\hline $\mathrm{M}_{3} \mathbf{S}_{1}$ & $0.13^{\mathrm{ef}}$ & $0.40^{\text {ef }}$ & $0.47^{\mathrm{e}}$ & $1.13^{\text {ed }}$ & $0.31^{\text {dce }}$ & $0.74^{\text {dce }}$ & $0.72^{d}$ & $1.28^{\mathrm{cb}}$ & $0.22^{\text {cd }}$ & $0.57^{\mathrm{ecd}}$ & $0.60^{\mathrm{e}}$ & $1.21^{\mathrm{ed}}$ \\
\hline $\mathrm{M}_{3} \mathrm{~S}_{2}$ & $0.20^{\text {ed }}$ & $0.40^{\text {ef }}$ & $0.47^{\mathrm{e}}$ & $1.00^{\text {fe }}$ & $0.27^{\mathrm{dce}}$ & $0.55^{\mathrm{dfe}}$ & $0.70^{\mathrm{d}}$ & $1.12^{\mathrm{c}}$ & $0.24^{\mathrm{cbd}}$ & $0.48^{\text {ed }}$ & $0.59^{\mathrm{e}}$ & $1.06^{\mathrm{ed}}$ \\
\hline $\mathbf{M}_{3} \mathbf{S}_{3}$ & $0.20^{\mathrm{ed}}$ & $0.40^{\mathrm{ef}}$ & $0.47^{\mathrm{e}}$ & $1.00^{\mathrm{fe}}$ & $0.26^{\mathrm{de}}$ & $0.54^{\mathrm{fe}}$ & $0.70^{\mathrm{d}}$ & $1.10^{c}$ & $0.23^{\mathrm{cbd}}$ & $0.47^{\text {ed }}$ & $0.58^{\mathrm{e}}$ & $1.05^{\mathrm{ed}}$ \\
\hline $\mathbf{M}_{3} \mathbf{S}_{4}$ & $0.20^{\mathrm{ed}}$ & $0.27^{\mathrm{f}}$ & $0.53^{\mathrm{ed}}$ & $0.87^{\mathrm{f}}$ & $0.26^{\mathrm{de}}$ & $0.47^{\mathrm{f}}$ & $0.82^{\mathrm{d}}$ & $1.33^{\mathrm{cb}}$ & $0.23^{\mathrm{cbd}}$ & $0.37^{\mathrm{e}}$ & $0.68^{\text {ed }}$ & $1.10^{\mathrm{ed}}$ \\
\hline S.Em \pm & 0.02 & 0.06 & 0.09 & 0.09 & 0.07 & 0.08 & 0.11 & 0.14 & 0.04 & 0.07 & 0.09 & 0.09 \\
\hline Control & 0.80 & 1.33 & 1.80 & 2.20 & 0.87 & 1.53 & 1.70 & 2.0 & 0.83 & 1.43 & 1.73 & 2.10 \\
\hline S.Emt & 0.06 & 0.08 & 0.09 & 0.09 & 0.05 & 0.08 & 0.08 & 0.09 & 0.04 & 0.08 & 0.07 & 0.06 \\
\hline C.D. 0.05 & 0.18 & 0.240 & 0.27 & 0.28 & 0.15 & 0.24 & 0.24 & 0.27 & 0.12 & 0.23 & 0.21 & 0.19 \\
\hline
\end{tabular}

*means with same letters do not differ significantly under DMRT

Note: DAS - Days after sowing, SSNM- Site Specific Nutrient Management

Main treatments: Yield Target $(M)$

$\mathrm{M}_{1}$ - SSNM for targeted yield of 3 tha $^{-1}$

$\mathrm{M}_{2}$ - SSNM for targeted yield of 4 tha $^{-1}$

$\mathrm{M}_{3}$ - SSNM for targeted yield of 5 tha $^{-1}$

Control-RDF with recommended practice
Sub treatments: Leaf reddening management (S)

$\mathbf{S}_{1^{-}}$Vermicompost @ 2.5 tha $^{-1}$ in seed line

$\mathbf{S}_{2^{-}} \mathrm{S}_{1}+\mathrm{MgSO}_{4} 10 \mathrm{kgha}^{-1}$ in seed line

$\mathbf{S}_{3^{-}} \mathrm{S}_{1}+\mathrm{MgSO}_{4} 25 \mathrm{kgha}^{-1}$ in seed line

$\mathbf{S}_{4}-\mathrm{MgSO}_{4} 25 \mathrm{~kg} \mathrm{ha}^{-1}$ in seed line + foliar nutrition of $1 \%$

$\mathrm{MgSO}_{4}+19: 19: 19+1 \% \mathrm{KNO}_{3}$ (thrice each)
Productivity efficiency was significantly higher $\left(0.50 \mathrm{~kg} \mathrm{ha}^{-1} \mathrm{dm}^{-2} \mathrm{day}^{-1}\right)$ with $\mathrm{M}_{3} \mathrm{~S}_{4}$ in comparison with $\mathrm{M}_{1} \mathrm{~S}_{1}\left(0.31 \mathrm{~kg} \mathrm{ha}^{-1} \mathrm{dm}^{-2}\right.$ day $\left.^{-1}\right)$ or control $\left(0.32 \mathrm{~kg} \mathrm{ha}^{-1} \mathrm{dm}^{-2} \mathrm{day}^{-1}\right)$. The productivity efficiency could be considered as an effective major for screening cultivars/ LRM techniques or any other production interventions to evaluate performance, resilience or susceptibility for leaf reddening in cotton under any agro climatic condition. This index could be considered more effective because it extrapolates leaf reddening index to the whole photosynthetic surface from flowering till final picking as a contributing 
factor towards yield. It is advantageous over either leaf reddening index or leaf area index alone because it considers ultimate photosynthetically effective leaf area during the reproductive cycle, while leaf reddening index is a fixed stage variation while reddening is dynamic and dependent on prevailing rhizosphere and crop microclimate conditions, and leaf area indicates total leaf area irrespective of its colour mosaic. Whereas, productivity efficiency is a single value relating yield versus potential photosynthetic leaf area during reproductive period (Chimmad, 1989; Prabhakar, 1981; and Upperi and Kuligoud, 2011).

\section{References}

Chimmad, V.P., 1989, Physiological and biochemical investigations on leaf reddening in cotton genotypes. Ph.D. Thesis, Uni. Agric. Sci., Dharwad, pp. $1-420$.
Cotton Advisory Board (CAB), 2016-17. https://www.icac.org/econ/Sources-ofCotton-Statistics/Asia/South-Asia/India

Hebbar, K. B. and Mayee, C. D., 2011, Para wilt/sudden wilt of cotton - a perspective on the cause and its management under field condition. Curr. Sci., 100(1): 1654-1662.

Prabhakar, A.S., 1981, Agronomic investigations on irrigated hybrid cotton. Ph.D. Thesis, Univ. Agril. Sci., Bangalore, pp. 1-380.

Upperi, S.N. and Kuligoud, V.B., 2011, Effects of prolonged and integrated use of organics and inorganics on the performance of cotton. World Cotton Res. Conf. on Technol. for Prosperity 5, Mumbai, 7-11 November 2011, Book of Papers, P. 359-363.

Venkateshwaralu, K., 2002. The Hindu, December 30, 2002.

\section{How to cite this article:}

Vinayak Hosamani, B.M. Chittapur, Mallikarjun, A.S. Halepyati, Satyanarayana Rao, M.B. Patil, N.L. Rajesh and Venkatesh Hosamani. 2018. Productivity Efficiency and Leaf Reddening in Bt Cotton (Gossypium hirsutum L.) as Influenced by SSNM Based Nutrition for Targeted Yields. Int.J.Curr.Microbiol.App.Sci. 7(08): 4118-4124.

doi: https://doi.org/10.20546/ijcmas.2018.708.428 